\title{
Older Professionals' Adaptation to New Information Technologies: A Study in the Context of Non-Standard Employment
}

\author{
Christoph Niesel \\ School of Information Systems \\ Queensland University of Technology \\ christoph.niesel@qut.edu.au
}

\author{
Alireza Nili \\ School of Information Systems \\ Queensland University of Technology \\ a.nili@qut.edu.au
}

\begin{abstract}
Modern workplaces are increasingly reliant on information technologies (IT), which have begun to profoundly affect the technological adaptation behaviors of older workers. Despite globally increasing levels of professional older adult workforce participation and workplace technology use, research in this space is only emerging, particularly in the context of non-standard employment (NSE), work falling outside traditional labor agreements, increasingly adopted by older workers. Using the Self Determination Theory, this qualitative work aims to understand the factors that contribute to professional older workers adaptation to new ITs in NSE. We propose a theoretical framework to explain our findings, suggesting that older professionals' adaptation to new ITs in NSE are motivated by competence, relatedness and autonomy and moderated by perceived occupational value, IT support, IT-based frustration and generational differences. Our findings offer a comprehensive understanding of motivational dynamics in our research phenomena.
\end{abstract}

\section{Introduction}

A wave of digitization changes across the labor market in the last two decades have drastically affected modern labor, including rising levels of nonstandard employment (NSE). The rapid changes due to digitization in employment have also affected older workers, who form a large share of the modern workforce worldwide, particularly in North America, Europe, Australia, China and more so in Japan [1]. These older workers (identified as roughly between 55-75 years of age) are generally seen as working longer past traditional retirement age and increasingly in NSE-related capacities, despite their precarious and sometimes disadvantaged position in modern workforces [2], [3]. NSE is an umbrella term that encapsulates work falling outside traditional labor agreements, including the commonly encountered working arrangements of casual, part-time, contractual and temporary work [1].

Within an increasingly digitizing workplace, it is understood that modern NSE participation is increasingly reliant on the ubiquity of workplace ITs [1]. This is owing to an often more remote, less organizationally structured and different working space and environment for broad NSE arrangements, often more prevalent for professional white-collar roles [2]. Furthermore, in a period of escalating workplace IT diffusion, it is suggested that successful adaptation to new ITs are critical for organizational and worker efficiency and productivity [4]. As an increasing number of nations worldwide face transformation into dominantly ageing societies, understanding motivations for successful adaptation to new workplace ITs is a paramount concern for the often-discounted older worker. Also, understanding the older worker is important based on gaps in literature and generational differences with younger workers. Technological, environmental and agerelated considerations remain as demographical differentiators, [5] despite the fact that not all younger workers are technologically proficient and many older workers are, despite stereotypes, IT capable [6]. It is also understood that for many older workers, digital exclusion in a period of rapid IT workplace diffusion is a very real concern for both organizations and workers [3]. Successful IT adaptation for older workers is a key link in understanding future workplace participation, particularly NSE. This link is still missing as current literature does not often focus the modern older worker or 'Baby Boomer'. Research is only beginning to understand the IT-related concerns and behaviors of this demographic which forms a large segment of modern labor markets [7]. Overall, despite a multitude of studies in the Information Systems (IS) field concerning technology use in the workplace [8], [9], we identified an absence of literature focusing the trichotomy of adaptation to new ITs, older workers and NSE in IS literature. 
In IS research, adaptation behavior has been defined as the degree an IT user adapts actively to an IT by augmenting or even substantially altering his or her working habits, routines (task management or flow) and the IT itself to achieve greater productivity [10], [11]. An individual's use of an IT new to them may augment or even substantially alter their existing work practices [12]. In this study, adaptation is seen as users using a new IT in an NSE workplace. Unlike adoption, it may be seen in terms of a user faced with a new mandatory system at work or significant changes or upgrades to existing workplace ITs. According to Beaudry and Pinsonneault's Coping Model of User Adaptation [13], two major types of adaptation behaviors are proposed, including: emotion-focused and problem-focused adaptation. Emotion-focused adaptation concerns the regulation of emotions in response to an issue. These often involve coping mechanisms such as seeking social support, reappraising an IT positively, or avoidance [13]. Problem-focused adaptation seeks to manage issues related to an IT event by (1) personal adaptation, adjusting one's own habits or tasks to fit with a technological requirement, or learning or updating a new technical skill [12], [13]; (2) making changes to a work task to adapt to the IT [14]; and/or (3) adapting the IT by changing its functionalities [13]. These adaptation methods often lead to a deeper use of a system which can result in increased efficiency and effectiveness at work [11]. Although emotion-focused adaptation is important, we choose to focus problemfocused adaptation for two reasons. First, choosing to study one type of adaptation behavior enables an indepth understanding of the behavior. Second, our study aims to identify older professional workers motivations for actions that are directly related to adaptation behavior (e.g., adjusting work-related habits to fit the requirements of an IT) within which a problem-focused approach allows understanding of action oriented adaptation. Thus, this approach is effective in illustrating deeper levels of IT use and pragmatic adaptation strategies and outcomes.

To identify and explain problem-focused adaptation, we explain the choice of SelfDetermination Theory (SDT) as the broad theoretical perspective to support a grounded theory method of analysis, and explain how remaining open to insights emerging from our qualitative data supported us in identifying factors emerging from data. We offer an original theoretical framework as a unique contribution to the IS space in motivational IT use dynamics. We respond to calls for richer theorizing outside native IS theories [15], [16] while addressing an important demographic of workplace IT users to answer the research question: What are the factors that contribute to older professionals' problem-focused adaptation in response to new ITs in NSE? The findings add to our knowledge within the broad space of IS use behavior. More specifically, the findings contribute to both theory and practice by helping to better understand the digital work behaviors of older workers (specifically professionals) and the implications of the proliferation of ITs for these workers participating in a growing form of digitizing labor, NSE.

The following section provides an overview of studies within the space of NSE, older workers, and their IT use. We then discuss theoretical positioning, research method, and the findings. A theoretical framework is presented, with a discussion aiming to identify contributions to IS within our research phenomena.

\section{Overview of literature}

The share of older workers who seek or have been pushed into non-standard labor is increasing worldwide [1]. Reasons for engagement in NSE revolve strongly around financial pressures in older age, dwindling job security in traditionally stable forms of work, and sector specific digitization and automation [17]. These are countered by positive motivators for NSE engagement, namely a desire for continued labor participation for physical and mental stimulation, an inclination towards a more relaxed lifestyle that is dependent on remuneration beyond pension schemes, and emerging groups of older workers who are defining later life as time for further career development [17]. Although digitization has aided the spread of NSE labor opportunities, older workers are often more vulnerable in workplace relations owing to poor stereotypical perceptions of them as "digitally inept", while those who are digitally unengaged increasingly stand to be excluded from the modern workforce [6]. The impact of negative social perceptions has been proven to create a toxic cognitive overload in the minds of older workers, impacting their IT use behaviors [18]. More recent studies have found that age plays a prominent role in fostering ICT use by older workers, where job insecurity owing to digitization is a paramount concern [7]. Despite increasing levels of NSE engagement, many older workers fall on the wrong side of a "digital divide" and are often viewed as digital adaptors rather than natives, which has been exacerbated by a digitizing workplace, particularly in NSE [19], [20] . Such trends have begun a call for further IS research in a modern phenomenon of increasing importance [21]. 


\subsection{Older worker IT use}

Although numerous studies exist covering older adult IT use, fewer focus on older professionals' use of workplace ITs, and we did not identify any study that focuses on older professionals' adaptation to new IT in NSE. More specifically, we did not identify any IS study that focuses on motivations for adaptation to new ITs in NSE. There are, however, some interesting studies (although not fully relevant to our work). Some have highlighted the importance of social ties as motivators influencing job performance technology use [9], while others have found the importance of antecedents such as disposition to job and organizational relationships having a strong impact on lean and rich measures of systems use in the workplace [4]. Regarding motivations for older adult IT use, Hanson (2010) stresses the complex dynamic that is the interplay of interest in, experience of and perceived usability of ITs by older workers. Mitzner (2010) further identified strong motivations of older workers towards technology-supported activities, with perceived convenience and ease of use as fundamentally important. Although few studies have looked at motivations for older worker IT use, some have identified a strong 'need of use' of technologies as a principal use motivator [6]. These findings are strengthened with fundamental work by Selwyn (2004), who argued that technological disinterest by older workers is a result of general ambivalence towards ITs seen as unnecessary by the user in accomplishing a task. Technology use behaviors in the workplace are also affected by prior workplace technologies that have been used by or exposed to older workers. These prior use attitudes, behaviors and motivators are largely seen to carry on to older age within and without the workplace [8], [22], and likely carry to attitudes towards technologies in NSE.

Overall, there first appears to be a lack of studies focusing on a younger group of older workers (conceptualized in literature as approximately 53-70 years old [21]), those who make up a large segment of the older workforce. As a result, we find less relevance in this work with the cognitive decline and later life age related changes that are often reported in older adult technology use studies as fundamental barriers to use [5], [23]. Second, a vast majority of IS studies in this space focus on the adoption of technologies (similar to TAM studies) [24] by older workers rather than on their motivations for adaptation (e.g. adjusting work-related habits to fit the requirements of the IT, modifying a task to fit functionalities of an IT, and learning new skills). Thus, the nature of the modern older worker, as a user rather than an adopter, remains largely unexplored [21].

\subsection{Theoretical perspective}

SDT is a prominent psychological theory using the premises that natural progression for humans veers to an inclination for psychological growth and internalization, and that people will affect and be affected by the environment around them, which can both support and obstruct such progression [25]. SDT has been used extensively since its inception in 1985, with iterative developments having occurred across social psychology, health and education, though few IS research studies have used it [25]-[27]. SDT posits three innate psychological needs of competence, autonomy and relatedness which, when satisfied, spur motivated action [27]. Competence denotes a sense of ability in task or action; autonomy relates to an individual's sense of choice in self-initiation or selfregulation (management) regarding behavior; and relatedness refers to a sense of belonging or closeness to others. SDT also suggests that intrinsic motivation is affected by external forces, for example, events which strengthen an individual's perceived competence will likely aid in strengthening intrinsic motivation. These include external events like rewarding productivity at work through financial incentive [26], positive or negative technology performance expectancies at work for specific tasks [28], and frustration-causing events in workplaces [29], in keeping with the context of this study. Loss or deterioration of these needs creates amotivation.

Building off these three innate needs, SDT plots three main motivational states across a continuum, amotivated, extrinsic and intrinsic. Across these three states, the theory proposes that individuals may integrate any form of goal or value in relation to a state of motivation at any given time, based on their own internalization of these behaviors. The continuum moves through levels of personal internalization (taking in a value or goal, i.e. learning a new system) of behaviors to a level of intrinsic motivation that is fully internalized and self-integrated [25]. Extrinsic regulation motivations are varied and include 1) external regulation, where individuals' motivations are controlled owing to factors that force them to act (and may include external rewards or punishments), such as financial incentive or deadline penalties at work; 2) introjected regulation, where individuals engage in behavior based on negative or positive impacts on their ego or self-worth (i.e. work task completion increasing personal confidence) and; 3 ) identified regulation, where behavior is endorsed or in line with an individual's values (i.e. creating a green space in an office aligns with environmental values). It is important to clarify that the SDT model allows 
individuals to move through this continuum in as they externalize or internalize behaviors [27].

Although not often used independently in IS, some studies have argued the importance of autonomy, competence and relatedness, which are often left unstudied alongside other IS theories [28]. SDT, however, has been used at a large scale in employment and gerontological studies to better understand motivation in work related task engagement and use behavior. These include support for claims that the fulfillment of the three innate psychological needs in workplaces directly contributes to intrinsic motivation [26], as well as the importance of financial incentive and strong managerial support as external moderators in promoting intrinsic work motivation [29]. SDT was also used to demonstrate the work motivation of older workers in pre-retirement, demonstrating that low intrinsic motivation was related to gains in autonomy satisfaction, whereas high intrinsic motivation was related to relatedness satisfaction [30].

\section{Method}

An inductive qualitative approach was undertaken, using semi-structured interviews. This was owing to the nature of our research phenomena, wherein a better understanding of the contributing factors to our research phenomenon was tied to deep levels of understanding from our participants.

We recruited participants through online advertisements (recruitment media releases through our university), by engaging with local organizations (e.g non-profit organizations supporting older professionals, local temporary worker groups) and through cold-contact email such as LinkedIn using snowball sampling. Despite some criticisms of bias in snowball sampling [31], this method was adopted as it afforded greater initial access to more niche participants (in NSE, a specific age range and using new ITs for work). Recruitment occurred in the state of Queensland in Australia. Our requirements were that participants were aged between 53 and 70 at the time of recruitment (moving into and just passed pension qualifying age in Australia) and working in NSE (we focus more commonly encountered forms of NSE casual, part-time, and contractual work). An open-ended set of requirements allowed for inclusive capture of a strong cross-section of the participant demographic. Overall, 25 participants were interviewed and comprised 17 men and 8 women from a variety of backgrounds including consultants, authors, academics, board-members and software developers. We achieved theoretical saturation and depth of insights with 25 interviews, commensurate with sample sizes of similar qualitative works [6], [8],
[17], [32]. We therefore stopped conducting new interviews at this point

Interviews were between one and one and a half hours in length and were conducted by the lead author in a variety of settings including homes, workplaces, and cafes. An interview protocol was designed and reviewed by three academics whose expertise lay in older adult workplace technology use. Interview questions were developed around seeking to understand different types of adaptation behaviors being exhibited by participants, motivations, facilitators and barriers for adaptation and the influence (if any) of the context of being an older worker in NSE. The suitability of the interview questions was validated by three academics who have direct and relevant research interest to the topic of this study. Among other questions, the protocol asked interviewees to: 1) identify newly used workplace ITs; 2) express opinions about their adaptation to these ITs; 3) explain their adaptation motivations; 4) to compare their adaptation to younger colleagues at work and to 5) explain what they felt aided in successful and unsuccessful adaptation of these ITs. The semistructured nature of our interviews allowed for emerging ideas or issues to be followed up and as a result, facilitated full exploration of participants' thoughts. This study was approved by an Australian university ethics and integrity committee. Data were de-identified and collected after participants' verbal and written consent obtained for research use. All interviews were audio-recorded and transcribed.

We adopted an inductive approach to data analysis, using the constructs of SDT as a guide to categorize emergent codes. We used grounded theory method, as SDT constructs are broad and while aiming to be open to concepts within their domain, we also wished to be open to insights and codes emerging from data [33]. This is in keeping with Burton-Jones \& Volkoff (2017), in allowing existing theoretical concepts to be used as a basis to create inductive theory while remaining open to new concepts [34]. The use of an existing broad theoretical basis with grounded theory is also in keeping with Layder's (1998) suggestions on theory creation [35].

Interviews were read multiple times and coded with Nvivo software. We undertook open, then axial and selective coding, wherein the latter two were conducted with SDT constructs in mind. Our approach allowed for new insights to emerge while being conscious of the broad constructs in SDT. Theoretical sampling occurred after the comments from the first 16 participant interviews were open coded, but we conducted more interviews (until the $25^{\text {th }}$ interview) to ensure saturation and coverage of ideas and themes. Axial coding then allowed for a breakdown of codes 
and concepts to be compared, where selective coding next allowed for themes and relationships to be identified. Several of our final themes were defined as factors that directly contribute to older professionals' problem-focused adaptation to new ITs in NSE. These themes are related to the constructs offered in our theoretical approach and are in keeping with the constructs of relatedness, competence and autonomy, with moderating themes (facilitators and inhibitors). The finding also included themes that moderate the influence of these factors on the research phenomenon.

Our data analysis placed importance on including a strong set of checks to ensure validity and reliability of research. Inter-coder reliability [36] was used for data coding and analysis, allowing authors to check any instances of bias formulating in the coding stages and ensure unanimous consensus was reached to control analytical interpretations. Checks were undertaken at each round of coding, with all authors agreeing on all factors. External validation was also sought by sending findings to participants, who confirmed the results as reflective of the information given in interviews. We include raw interview excerpts to allow for full transparency of the work.

\section{Findings}

Despite some variance in labor arrangements, participants were broadly using ITs in their workplace which included (but were not limited to) job-specific productivity tools (AutoCAD, Adobe suite, Xero account software, Myob, Zoho training software) and tools such as Outlook, social media and blogs. Participants were conducting their work on desktop and laptop computers, smartphones, and sometimes tablets. Although some of these ITs were not new to market, our participants were new to using them and therefore adapting. Some users adapted themselves to these new ITs and some did not. We aimed to understand why.

To answer our research question, we identified several factors, organized into themes. These include (a) three themes related to competence, autonomy and relatedness, each of which directly contributes to the research phenomenon (older professionals' adaptation to new IT in NSE); and (b) two other themes, labeled as facilitators and inhibitors, each of which moderates the effects of the three themes on the research phenomenon. These findings together constituted our theoretical framework.

\subsection{Factors that directly contribute to older professionals' adaptation to new ITs in NSE}

These themes correspond to the three main constructs of SDT, competence, autonomy and relatedness in the context of our study. We explain each below.

Competence: Perceived competence at work. We realized that a sense of perceived competence at work contributes to adaptation to new ITs. Although perceived competence at work was individually varied, the majority of participants were motivated by the belief that they were good at their job. This often led to an inherent enjoyment of work tasks (intrinsic motivation) that supports tendencies to adapt to IT, demonstrated with this quote.

"I've been doing what I do for a while now, so I think I'm decent at my work. It helped me feel confident when I transitioned to working at home. It doesn't mean I'm amazing at the tech stuff, but that doesn't worry me much, you know?"

This relationship is exemplified in a quote by the below participant, a consultant, who stated that:

"I am fortunate that I actually enjoy work, growing companies, I enjoy it and am good at it. I have been successful a couple of times and it gives you a big kick. I can't imagine reaching 65 and saying, "That's it," because I enjoy it."

Competence: Perceived competence at modifying task characteristics and IT functionalities. The participants felt that there was a strong need to be adaptation competent, owing to ongoing technological diffusion. To adapt to new ITs, participants expressed the tendency for modifying their existing work tasks and changing ITs' functionalities to suit their work. The majority expressed a higher level of competence and tendency in the former. Work task modification was an external motivation based on perceived need of using the IT for their work (identified regulation) and such adaptation was reported as a means to an end. For example, one participant illustrates this viewpoint.

"I have polished my LinkedIn profile and as recently as yesterday, I am learning how to use that. Because when I talk to my younger business advisors they say, "Social media". I need to change the way I work to fit the current era and the tech tools that I now use."

Autonomy: Perceived control in work-life balance. Participants unanimously expressed strong levels of perceived control in their work-life balance, made accessible by working in different forms of NSE. 
While IT use facilitated this (such as remote offices), the sense of freedom provided by NSE working styles and (to an extent) a reduction of traditional workplace organizational constraints were an intrinsic motivation for the levels of perceived control they had in their working lives, as exemplified in the quote below.

"It is kind of handy to have your workplace set up with the resources that you need at your fingertips. My style of work provides autonomy"

Autonomy: Perceived control in adaptation to workplace ITs. Autonomy was viewed under the theme of perceived control, with participants expressing various levels of control in adapting to a new workplace IT. New ITs were introduced with frequency, often seen as necessary and motivated by organizational pressures (external regulation), with participants seeking to sometimes make changes to work tasks to adapt to an IT. Limited perceived control directly affected participants difficulties in accomplishing work tasks, as mentioned below.

"I'd never worked with Google to share stuff. And in our little organization, they just assumed you understood it. I tried to adapt to the new Mac programs and they just didn't cut it for me, because I had always been weaned on Microsoft Word and Excel, so they never did what I wanted them to do, which was hard."

Relatedness: Strengthening work-related social relationships. Participants were motivated by a desire to maintain and foster social relationships through use of new ITs. These were mainly intrinsic motivations, though sometimes extrinsic and based on personal values of socialization (identified regulation) or ego (introjected regulation). IT adaptation was sometimes a pathway to strengthen work-related relationships, often relevant for communication or socialization centric ITs. Social relationships were important in NSE, where participants (sometimes working remotely) were often more reliant on ITs for maintaining work-related relationships. Relatedness was relevant when participants were adapting new ITs as part of a team or organization, where a new IT became a point of conversation or socialization. For example, a participant who recently moved into NSE reported:

"The great thing is adapting some of these new social networking sites for business. Moving into part-time work gave me a home office, but see, it was rather hard to maintain social contact with clients and friends I'd previously worked with, before I made the transition."

\subsection{Moderating themes}

We identified facilitators and inhibitors as two themes moderating the influence of the previous themes for adaptation to new ITs. As participants' comments show, these facilitators and inhibitors moderate (i.e. can influence or change the degree of) the effect of the other three constructs (relatedness, competence, and autonomy) on the research phenomenon.

Facilitators. We identified two facilitators: perceived occupational value and IT support, explained below.

Perceived occupational value. Participants demonstrated that perceived occupational value can influence the strength of their motivations for adaptation to new ITs. Specifically, we realized that perceived occupational value facilitates motivational disposition (extrinsic and intrinsic) towards adaptation to a new IT. The quote below illustrates this influence and personal adaptation.

"I'm on LinkedIn for work and I have to say, I only went on there probably, within the last year. And that's partly because when I was asking around, you know, "How do you get clients?" and so on, people were saying, "You have to be on LinkedIn".

IT support. Availability of support in adapting a new IT aided in fostering confidence for participants, bolstering competence and autonomy. We identified two forms of support which included vendor/IT helpdesk support and self-help resources (for example, FAQ's, text-based user manuals and video instructions). Vendor/IT help-desk support was particularly prevalent in the case of NSE, where participants felt greater support aided in their work, which was traditionally seen as offering fewer instances of support for IT (mainly for participants working within a looser organizational structure). Where such support was not available or where participants were seeking for straightforward solutions, self-help resources played an essential role. For example, a participant mentioned:

"If I need to find something related to the new technologies we use at work, I don't need to go much further than jumping online. A quick Google search usually yields anything I need and if not that, most basic things exist as tutorial videos." 
Inhibitors. We identified four inhibitors including: IT based frustration, cost of time investment, financial cost, and generational differences related to effort for adaptation. We explain these factors below.

IT-based frustration. An expectation for new ITs to work without hassle was expressed as a factor that influenced participants' adaptation motivations. Participants in NSE often used multiple ITs to accomplish work tasks, leading to an expectation that technologies would be frustration-free, shared below.

"A trouble would be the frustration that I create with when something new turns up. You expect to plug it in and make it work."

Cost of time investment. New technologies were related to a cost in time investment for learning a new skill or updating current skills to use them, which often saw participants' motivations for adaption to decrease. For example, a lack of time investment led to less perceived competence because participants were less likely to feel confident about using a new IT without investing time into it.

"Look, I know most of the things I need for work and though some new software looks useful, I just don't know if it's worth my time adapting to something new which may or may not be handy."

Financial cost. Although many ITs were mandatory/organizationally required, expenses were still associated with their adaptation in the NSE context. Most participants were hesitant about such expenses. This was a complex issue for NSE participants, who often had multiple competing systems available for purchase to accomplish work tasks. High financial cost impacted motivations for adaptation, with the quote below explaining diffusion of ITs across business platforms.

"From a small business point of view, there's so many new platforms. The problem is when you start building them up, they start getting expensive. When you are paying $\$ 50$ for this platform and $\$ 50$ a month for that software, it adds up."

Generational differences related to effort for adaptation. Although many participants had demonstrated a general understanding of IT use in the workplace in their profession, their adaptation motivations were still moderated by their perception of generational differences. Most participants reported little desire in expending effort for adaptation to new
ITs, owing to their belief that they are not young users. For example, a participant expressed a sense of difficulty in expending effort for adapting to ITs in the workplace because of older age:

"I sometimes feel, because I'm not born in this time, I'm a bit "thumby". I get there but I get there eventually. I think if I was born 20 years later, it (my IT use) would be more second-nature."

Another participant, who showed a moderate level of desire for personal adaptation, stated:

"I'm not technophobic because of the technologies used in my previous work but I'm not techno-savvy. I would love to have that knowledge, but as a non-digital native, and despite being someone who likes technology, I don't like it enough to spend hours trying to understand it."

Figure 1 is our theoretical framework, illustrating the factors that contribute to older professionals' problem-focused adaptation to new ITs in NSE.

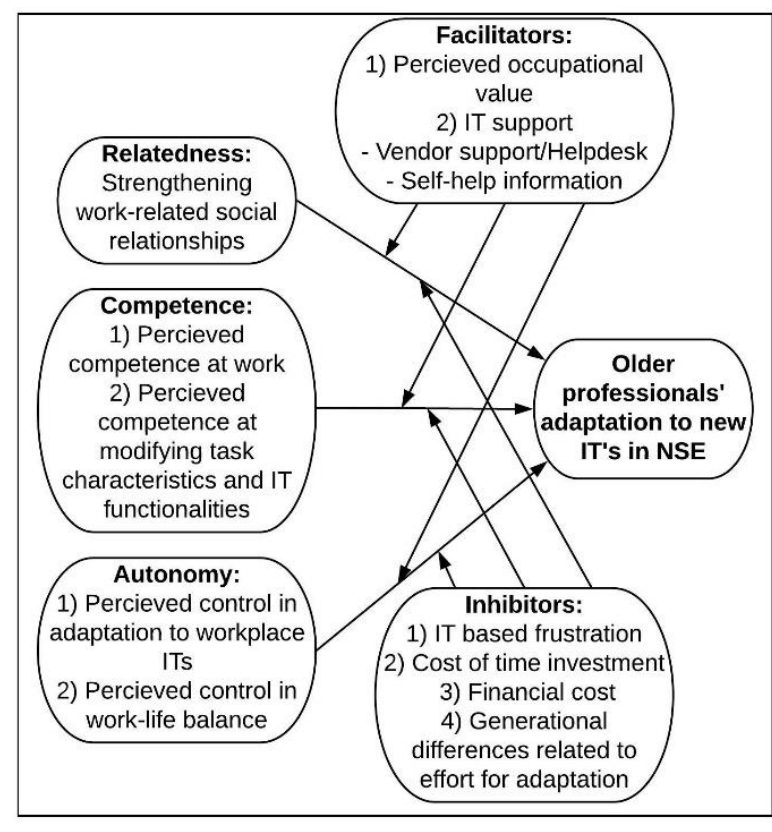

Figure 1. Theoretical framework of motivational and moderating themes for older professionals' adaptation to new ITs in NSE.

\section{Discussion}

Our work offers novel findings that provide a better understanding of the factors that contribute to 
older professionals' adaptation to new ITs within the NSE context, and the impact this has on the proliferation of work-related technologies at a time of an increasingly ageing workforce. This study has extended SDT within our research phenomena and in the IS discipline. We identified motivations and moderators with regards to problem-focused adaptation (our IS use behavior research phenomena), offering an original perspective of older professionals in NSE (our participants backgrounds sensitized us that they were, specifically, white collar professionals and thus we term them in our work). Regarding the factors that directly contribute to the research phenomenon, relatedness was governed mainly by intrinsic motivations to socialize in a work context (particularly in NSE), while the factors within the competence and autonomy themes contain mixed motivational sources. Interestingly, intrinsic motivations were present at greater levels within purely work-related factors such as competence at work and strengthening social relationships, whereas extrinsic (often strongly externally regulated) motivations were found in IT-related factors, such as perceived control in IT adaptation. This may suggest IT related activities at work are often not a point of inherent interest for older professionals. Broadly, NSE workers appeared to be more reliant on workplace ITs than those in full-time roles [1], [2]. Our findings suggest that for many older professionals faced with adaptation to new ITs in the workplace, a need to adapt to these ITs is an initial extrinsic motivation and this adaptation is almost mandatory owing to the necessity of specific workplace ITs, even in NSE.

The proposed theoretical framework also illustrates facilitators and inhibitors unique to our specific context. Perceived occupational value demonstrated that although mandatory ITs exist for many older professionals in NSE, the greater latitude and control of work afforded to them through NSE participation often translated to greater choice in selecting ITs which fulfill their work needs. Although this still meant an IT supported task was often necessary in their workflow, the choice of IT to support that task was often broader and less prescribed. Access to IT support was also perceived as important in an NSE context, owing to the sometimesinfrequent organizational support structures offered to those working at home offices or remotely. Although our inhibitors were consistent with existing IS literature identifying IT frustration [15] and time investment cost [5] as deterrents, we found that financial cost was more unique to NSE, as workers were more likely to use personal funds to partly finance their work. Moreover, the moderator of age in relation to effort for adaptation within our theory is understood to be an important inhibitor. This is commensurate with literature based on older adult technology use [6], [18], [23]. Despite some earlier suggestions that a younger cohort of older professionals (those who have received greater IT related training in the workplace at a younger age) may demonstrate fewer barriers for adaptation related to age [37], we have found that most older professionals still harbor perceived generational differences, though this perception may be unfounded in practice. We find that although many participants were familiar with fundamentally "basic" workplace technologies (Microsoft Word, Outlook etc.) and somewhat technologically proficient, adaptation was still seen as a "young person's game" requiring membership as a digital native.

In implications for practice, we propose the importance of occupational value of using ITs and access to help as the main facilitators of IT adaptation for older professionals and that organizations pushing for new ITs must be wary of the multitude of costs (time, frustration, financial) that can be associated with their adaptation. Although such recommendations align with the aims of most organizations, older professionals' considerations highlight the nature of these issues as more acute fundamental barriers for IT adaptation, exacerbated by perceived generational gaps. In practice, age-diverse cooperation in industry may benefit older professionals [7]. Intergenerational training and cooperative work may aid in fulfilling a desire for IT support (a facilitator in our work), as well as tightening a perceived digital divide. Implementing intergenerational cooperation however can be difficult in NSE, owing to a sometimes less physically tangible organizational structure or environment in the workplace. We suggest that organizations with a share of older professionals actively in NSE can give, where possible, extra levels of IT support, including greater peer-support by offering platforms, online discussion forums for staff, and producing online training resources. Findings also demonstrated that strong extrinsic motivations of competence and perceived control at adaptation to new ITs often-pushed older professionals to continue integrating ITs in their work. If organizations can highlight and clearly demonstrate the occupational values of new ITs to workers, this may ease and quicken adaptation.

This work furthers knowledge of adaptation use behavior in IS research, focusing an emergent and timely group of older professionals, a focal demographic over coming years. By using the grounded theory approach, we brought the SDT theory (a theory with broad constructs) to our study context. We contextualized each of its three constructs by 
identifying relevant dimensions, then extended the theory through adding two types of moderators: facilitators and inhibitors of older professionals' adaptation to new workplace IT. We realized that older professionals are personal adaptors, who tend to adjust their work habits or tasks for the IT, over other adaptation behaviors. Though many participants modified work tasks in response to an IT, few adapted the IT by changing its functionalities, suggesting low competence in this area, which is also affected by perceived generational differences for adaptation (one inhibiting factor). What must be emphasized however is that lower instances of effort related to adaptation does not necessarily indicate digital ineptness, an often prevalent stereotype [18]. This work also offers a wider perspective in use behavior with the SDT, suggesting effort expended for new IT adaptation may manifest from the core values of relatedness, competence and autonomy, guided by our identified facilitators. For older professionals in NSE, ITs seen as lacking in workplace value or coming at high cost(s) are likely to be detrimental to motivation. Overall, though we offer new insights within a unique research phenomena, our findings and suggestions align with existing research in this emerging space, suggesting that older professionals do harbor some age related differences in the workplace [22], that motivations are often based around perceived personal ability [6], [32] and that perceived IT workplace value strongly influences such motivations [7].

\section{Limitations and conclusion}

Our work has some limitations. Our participants came from a generally higher socio-economic bracket, the majority holding strong education and training backgrounds, thus labeled older professionals. We also had a lower ratio of women to men $(8: 17)$, despite inclusive participant recruitment. NSE is also broad and while we focused on the more broadly represented forms of work within, a case-by-case study of adaptation behaviors in different forms of NSE may reveal further insights.

For future work, a greater balance of gender in participants and longitudinal studies may expand adaptation behavior findings. Moreover, the significance of cost as a primary inhibitor in our work suggests that cost-focused theoretical approaches such as expectancy-cost-value may unearth greater inhibitor-specific findings. Finally, we suggest a focus on emotion-focused adaptation as a worthwhile area of future research, to fully capture adaptation motivation.

We discussed the important timing of this work, owing to labor market ageing, increasing digital reliance during widespread workplace technological diffusion and rising levels of NSE for older professionals. To understand use behavior, or in this case adaptation, is to understand motivation, an aspect of IS research that cannot be understated if organizations and workplaces seek efficient working practices by digitally reliant older professionals in sometimes unstable forms of employment [3]. We identified the factors that contribute to older professionals' problem-focused adaptation in response to new ITs in NSE. By understanding these factors and their relationships to our research phenomena, we offer a more complete picture of adaptation behaviors for older professionals in a time of significant population ageing and growing demand for NSE.

\section{References}

[1] International Labour Organization, Non-standard employment around the world: Understanding challenges, shaping prospects. Geneva: International Labour Organization (ILO), 2016.

[2] W. Eichhorst and P. Marx, Eds., Non-Standard Employment in Post-Industrial Labour Markets: An Occupational Perspective. Edward Elgar Publishing, Cheltenham UK, 2015.

[3] C. Muntaner, "Digital Platforms, Gig Economy, Precarious Employment, and the Invisible Hand of Social Class," Int J Health Services, vol. 48, no. 4, 2018, pp. 597-600.

[4] H. Bala and A. Bhagwatwar, "Employee dispositions to job and organization as antecedents and consequences of information systems use," Information Systems Journal, vol. 28, no. 4, 2018, pp. 650-683.

[5] T. L. Mitzner et al., "Older adults talk technology: Technology usage and attitudes," Computers in Human Behavior, vol. 26, no. 6, 2010, pp. 1710 1721.

[6] R. Hill, L. R. Betts, and S. E. Gardner, “Older adults' experiences and perceptions of digital technology: (Dis)empowerment, wellbeing, and inclusion," Computers in Human Behavior, vol. 48, 2015, pp. 415-423.

[7] E. Soja and P. Soja, "Fostering ICT use by older workers: Lessons from perceptions of barriers to enterprise system adoption," Journal of Enterprise Information Management, ahead-of-print, 2020.

[8] M.-K. Stein, R. D. Galliers, and M. L. Markus, "Towards an understanding of identity and technology in the workplace," Journal of Information Technology, vol. 28, no. 3, 2013, pp. 167-182.

[9] X. Zhang and V. Venkatesh, "Explaining Employee Job Performance: The Role of Online and Offline Workplace Communication Networks," MIS Quarterly, vol. 37, no. 3, 2013, pp. 695-722.

[10] A. Bhattacherjee and M. Harris, "Individual Adaptation of Information Technology," Journal of 
Computer Information Systems, vol. 50, no. 1, 2019, pp. 37-45.

[11] K. J. Fadel, "User Adaption and Infusion of Information Systems," Journal of Computer Information Systems, vol. 52, no. 3, 2012, pp. 1-10.

[12] W. J. Orlikowski, "Using Technology and Constituting Structures: A Practice Lens for Studying Technology in Organizations," Organization Science, vol. 11, no. 4, 2000, pp. 404428.

[13] A. Beaudry and A. Pinsonneault, "Understanding User Responses to Information Technology: A Coping Model of User Adaptation," MIS Quarterly, vol. 29, no. 3, 2005, pp. 493-524.

[14] M. J. Tyre and W. J. Orlikowski, "Windows of Opportunity: Temporal Patterns of Technological Adaptation in Organizations," Organization Science, vol. 5, no. 1, 1994, pp. 98-118.

[15] P. S. Galluch, V. Grover, and J. B. Thatcher, "Interrupting the Workplace: Examining Stressors in an Information Technology Context," Journal of the Association for Information Systems, vol. 16, no. 1, 2015, pp. 1-47.

[16] M. Tate, J. Evermann, and G. Gable, "An integrated framework for theories of individual attitudes toward technology," Information \& Management, vol. 52, no. 6,2015 , pp. 710-727.

[17] E. Kojola and P. Moen, "No more lock-step retirement: Boomers' shifting meanings of work and retirement," Journal of Aging Studies, vol. 36, 2016, pp. 59-70.

[18] M. Noeltner, J. Kroenung, and B. G. Kuhlmann, "Disarming Prejudice: How Ease of Use Mitigates the Detrimental Effect of IT-Based Stereotype Threat on the IT Task Performance of Older Adults," in International Conference on Information Systems, Munich, 2019, p.17.

[19] C. Ball, J. Francis, K.-T. Huang, T. Kadylak, S. R. Cotten, and R. V. Rikard, "The Physical-Digital Divide: Exploring the Social Gap Between Digital Natives and Physical Natives," J Applied Gerontology, vol. 38, no. 8, 2019, pp. 1167-1184.

[20] B. Niehaves and R. Plattfaut, "Internet adoption by the elderly: employing IS technology acceptance theories for understanding the age-related digital divide," European Journal of Information Systems, vol. 23, no. 6, 2014, pp. 708-726.

[21] S. Tams, V. Grover, and J. Thatcher, "Modern information technology in an old workforce: Toward a strategic research agenda," The Journal of Strategic Information Systems, vol. 23, no. 4, 2014, pp. 284-304.

[22] T. E. Rizzuto, "Age and technology innovation in the workplace: Does work context matter?," Computers in Human Behavior, vol. 27, no. 5, 2011, pp. $1612-1620$.

[23] V. L. Hanson, "Influencing technology adoption by older adults," Interacting with Computers, vol. 22, no. 6, 2010, pp. 502-509.

[24] F. D. Davis, "Perceived Usefulness, Perceived Ease of Use, and User Acceptance of Information
Technology,” MIS Quarterly, vol. 13, no. 3, 1989, pp. 319-340.

[25] R. M. Ryan and E. L. Deci, "Self-determination theory and the facilitation of intrinsic motivation, social development, and well-being," American Psychologist, vol. 55, no. 1, 2000, pp. 68-78.

[26] E. L. Deci, A. H. Olafsen, and R. M. Ryan, "SelfDetermination Theory in Work Organizations: The State of a Science," Annual Review of Organizational Psychology and Organizational Behavior, vol. 4, no. 1, 2017, pp. 19-43.

[27] M. Gagné and E. L. Deci, "Self-determination theory and work motivation," Journal of Organizational Behavior, vol. 26, no. 4, 2005, pp. 331-362.

[28] Y. Lee, J. Lee, and Y. Hwang, "Relating motivation to information and communication technology acceptance: Self-determination theory perspective," Computers in Human Behavior, vol. 51, 2015, pp. 418-428.

[29] A. Van den Broeck, D. L. Ferris, C.-H. Chang, and C. C. Rosen, "A Review of Self-Determination Theory's Basic Psychological Needs at Work," Journal of Management, vol. 42, no. 5, 2016, pp. 1195-1229.

[30] G. Henning et al., "Preretirement Work Motivation and Subsequent Retirement Adjustment: A SelfDetermination Theory Perspective," Work Aging Retire, vol. 5, no. 2, 2019, pp. 189-203.

[31] B. L. Berg and H. Lune, Qualitative research methods for the social sciences, Pearson, Boston, 2012.

[32] N. Selwyn, "The information aged: A qualitative study of older adults' use of information and communications technology," Journal of Aging Studies, vol. 18, no. 4, 2004, pp. 369-384.

[33] A. Strauss and J. M. Corbin, Grounded Theory in Practice. SAGE, 1997.

[34] A. Burton-Jones and O. Volkoff, "How Can We Develop Contextualized Theories of Effective Use? A Demonstration in the Context of Community-Care Electronic Health Records," Information Systems Research, vol. 28, no. 3, 2017, pp. 468-489.

[35] D. Layder, Sociological Practice: Linking Theory and Social Research. SAGE, Great Britain, 1998.

[36] A. Nili, M. Tate, A. Barros, and D. Johnstone, "An approach for selecting and using a method of intercoder reliability in information management research," International Journal of Information Management, vol. 54, 2020, p. 102154.

[37] T. Broady, A. Chan, and P. Caputi, "Comparison of older and younger adults' attitudes towards and abilities with computers: Implications for training and learning," British Journal of Educational Technology, vol. 41, no. 3, 2010, pp. 473-485. 